\title{
BMJ Open Study protocol of the ACCESS trial: a randomised trial to evaluate the effectiveness of human papillomavirus testing by self-sampling in cervical cancer screening uptake and precancer detection
}

\author{
Misuzu Fujita (D) , ${ }^{1,2}$ Minobu Shimazu, ${ }^{3}$ Kengo Nagashima (D) , Misae Suzuki, ${ }^{5}$ \\ Ichiro Tauchi, ${ }^{5}$ Miwa Sakuma, ${ }^{5}$ Setsuko Yamamoto, ${ }^{5}$ Makio Shozu, ${ }^{6}$ \\ Hideki Hanaoka, ${ }^{3}$ Nobuhide Tsuruoka, ${ }^{7}$ Tokuzo Kasai, ${ }^{1}$ Akira Hata ${ }^{1,8}$
}

To cite: Fujita M, Shimazu M, Nagashima K, et al. Study protocol of the ACCESS trial: a randomised trial to evaluate the effectiveness of human papillomavirus testing by self-sampling in cervical cancer screening uptake and precancer detection. BMJ Open 2022;12:e049803. doi:10.1136/ bmjopen-2021-049803

- Prepublication history and additional supplemental material for this paper are available online. To view these files, please visit the journal online (http://dx.doi.org/10.1136/ bmjopen-2021-049803)

Received 23 February 2021 Accepted 15 January 2022

Check for updates

(C) Author(s) (or their employer(s)) 2022. Re-use permitted under CC BY-NC. No commercial re-use. See rights and permissions. Published by BMJ.

For numbered affiliations see end of article.

Correspondence to

Dr Akira Hata;

ahata@faculty.chiba-u.jp

\section{ABSTRACT}

Introduction Recently, the incidence of cervical cancer has increased in Japan, probably because of an interruption in human papillomavirus (HPV) vaccination and a low cervical cancer screening rate. There is a lack of evidence for self-sampling HPV testing as a cervical cancer screening tool in Japan. The Accelerating Cervical Cancer Elimination by Self-Sampling test trial aims to compare the effectiveness of screening using the self-sampling HPV test with that of routine screening concerning screening uptake and precancer detection. Methods and analysis This trial has a singlemunicipality, open-label, parallel, superiority and randomised design. Approximately 20000 women who have not undergone cervical cancer screening for at least 3 years will be assigned randomly to the self-sampling arm and the control arm using a 1:1 ratio. Participants assigned to the control arm will undergo routine cervical cancer screening (cytology test) provided by Ichihara City, while those assigned to the self-sampling arm will choose the routine screening or self-sampling HPV test. HPV tests will be performed using the cobas 8800 system (Roche Diagnostics, Rotkreuz, Switzerland). Participants who will undergo the self-sampling HPV testing will be recommended to undergo routine screening. The results of the cytology test and further tests, such as colposcopy and biopsy, will be collected and used for this trial. The risk ratio and risk difference in the proportion of participants with cervical intraepithelial neoplasia two or worse between the two arms will be calculated. The test for the null hypothesis (the detection rates are equal between the two arms) will be performed using Pearson's $\chi^{2}$ test. Ethics and dissemination This trial was approved by the Research Ethics Committees of the Chiba Foundation for Health Promotion and Disease Prevention and the collaborating research institutes. The results will be disseminated through peer-reviewed journals and conference presentations.

Trial registration number jRCT1030200276. Pre-results.
Strengths and limitations of this study

- This is the first randomised controlled trial to evaluate and compare the effectiveness of cervical cancer screening with self-sampling human papillomavirus (HPV) testing for non-responders to that of routine screening using real-world data in Japan.

- This trial will clarify whether screening using selfsampling HPV testing detects more cervical intraepithelial neoplasia 2 or worse than routine screening.

- This trial will provide suggestions about cervical cancer screening methods in Japan, regardless of whether the result is positive or negative, since it will use the most robust methodology.

- Analyses aimed toward implementation (eg, the participation rate of detailed tests, such as colposcopy and biopsy) and a questionnaire survey of the acceptance of screening using self-sampling HPV testing will also be performed.

- Generalisability might be limited because the participants of this trial are women living in one municipality in Japan.

\section{INTRODUCTION}

The predominant cause of cervical cancer is certain types of human papillomavirus (HPV) infection. Therefore, the best approach toward the prevention of HPV-caused cancer is by vaccination and screening. ${ }^{1}$

In Japan, the publicly funded HPV vaccination programme began in December 2010 for girls aged 12-16 years. However, in June 2013, the Ministry of Health, Labour, and Welfare suspended an active HPV vaccine recommendation due to reported adverse events. Thereafter, the vaccination rate in Japan decreased dramatically to almost $0 \%$ in $2015 .{ }^{2}$ Although 
preventive strategies for cervical cancer strongly depend on screening, the rate is extremely low in developed countries that are members of the Organisation for Economic Co-operation and Development, including Japan. ${ }^{3}$ These situations have increased the rates of mortality and morbidity due to cervical cancer, ${ }^{4}{ }^{5}$ especially among young women. ${ }^{5}$ In 2014, a total of 10490 cervical cancers were diagnosed in Japan, and there were 2790 cervical cancer-related deaths in 2017. The rates in the future are estimated to increase further. ${ }^{6}$ To prevent cervical cancer, effective screening systems to improve the screening rate are urgently required and should be implemented.

Unlike cervical cytology, the HPV test with a self-collected sample has a similar validity as that of a doctor-collected one. ${ }^{78}$ The sensitivity and specificity of the self-sampling HPV test to detect cervical intraepithelial neoplasia (CIN) two or worse are reportedly $92.9 \%$ and $93.9 \%$, respectively. ${ }^{7}$ The main reasons for women not opting to undergo cervical cancer screening are: 'a cytology test by a doctor is too embarrassing' or 'it is difficult to find time to undergo a cytology test. ${ }^{, 9-11}$ Self-sampling might be an alternative method to solve these issues in non-responders to screening. Indeed, previous studies conducted in the USA, ${ }^{12}$ Sweden, ${ }^{13}$ the Netherlands,,${ }^{14}$ Denmark $^{16}$ and France ${ }^{17}$ reported that the self-sampling HPV test increased the participation rate among nonresponders, and the test is already implemented in some countries as an option for non-responders. ${ }^{18} 19$ However, the current guidelines for cervical cancer screening in Japan do not yet recommend self-sampling HPV testing because of the lack of evidence for the test in Japan. The Japan Society of Obstetrics and Gynecology stated in 2019 that the society does not recommend self-sampling HPV test. Furthermore, they claimed that one of the requirements for approval of the self-sampling test would be to present concrete evidence in Japan, derived from studies that evaluate not only screening uptake, but also the consultation rate of detailed tests, such as colposcopy and biopsy. Thus, to implement a self-sampling HPV test in Japan, the effectiveness of the test should be evaluated using the most robust study design, a randomised controlled trial, in a real-world population.

The main objective of the Accelerating Cervical Cancer Elimination by Self-Sampling test trial is to evaluate and compare the effectiveness of screening with a selfsampling HPV test with that of routine screening in terms of screening uptake and precancer detection in Japan.

\section{METHODS AND ANALYSIS \\ Study design}

This is a single-municipality, open-label, parallel, superiority, randomised trial. Details are shown in the study protocol (online supplemental file 1).

\section{Eligibility criteria and randomisation}

Outlines of this trial and time schedule plan are shown in figures 1 and 2, respectively. The trial participants are women who lived in Ichihara City, Chiba Prefecture, Japan. The candidates for this trial will be selected from the database maintained by the Ichihara City Hall as of 22 December 2020, according to the following inclusion criteria:

- Women who lived in Ichihara City on 22 December 2020.

- Women aged between 30 and 59 years as of 1 April 2021.

- Target population for cervical cancer screening in Ichihara City in 2021 (women with an even number of age).

- Women who had not undergone routine cervical cancer screening in Ichihara City for 3 years or more.

Women with an incorrect address will be excluded. Other exclusion criteria will not be used, given the unavailability of relevant data in the database, such as marital status, sexual activity, and pregnant or lactating. Each participant's data administered by the city, including name, address, birthday, and latest year they underwent cervical cancer screening, will be provided to the Chiba Foundation for Health Promotion and Disease Prevention (hereafter, the Foundation), where data management for this trial will be carried out. On 23 December 2020, the inclusion criteria for each candidate will be confirmed, and the candidates for this trial will be registered. On 1 February 2021, we will send a preinvitation letter to all candidates who meet the inclusion criteria, which indicates that they can refuse to participate in this trial (opting out), as shown in online supplemental file 2. Moreover, the objectives and methods of this trial will be publicly available in a public relations newsletter from Ichihara City, a website of the city, and a website of the Foundation on the same day the preinvitation letter will be sent. On 22 February 2021, women whose preinvitation letter returned due to incorrect address or women who opted out by 19 February 2021, will be excluded from the trial. The remaining women will be enrolled in this trial and randomly assigned to the self-sampling arm and the control arm using a 1:1 ratio, according to computer-generated random numbers. All procedures of assignment will be performed at the Foundation at once (central randomisation). The participants who will be assigned to the self-sampling arm will be informed in the second invitation letter. Meanwhile, the participants who will be assigned to the control arm will not receive any information regarding this trial after assignment, which will be stated in the pre-invitation letter. It will not be possible to blind the assignment to participants and screening providers (researchers) due to the nature of the intervention.

\section{Procedures in each arm}

Control arm

The city offers routine cervical cancer screening, cytology tests, every 2 years to women aged 20 years or more living in the city. In the fiscal year 2021 (between 1 April 2021 and 31 March 2022), the target population 


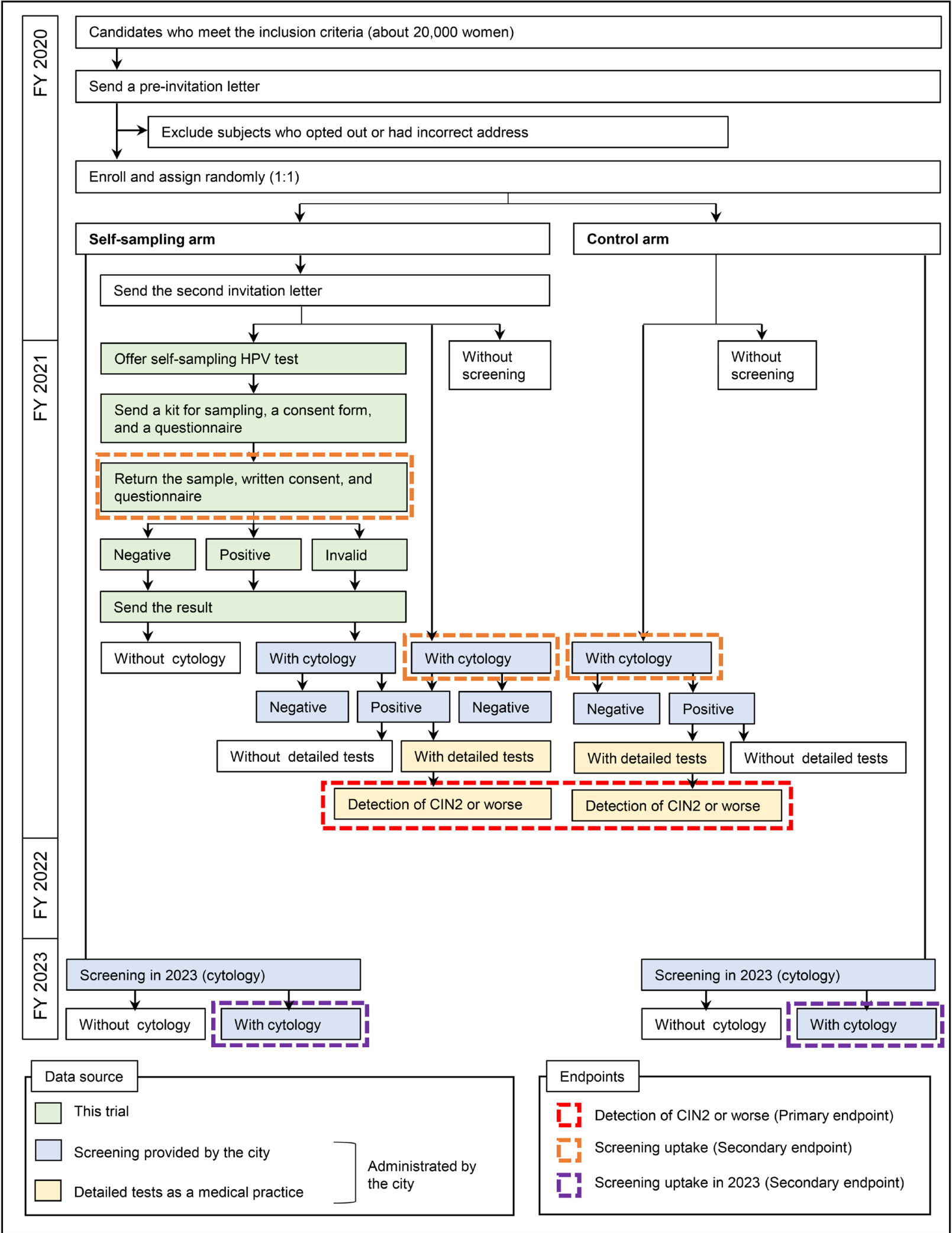

Figure 1 Flow chart of the study protocol. CIN2, cervical intraepithelial neoplasia; HPV, human papillomavirus.

for the screening will include those with an even number of age as of 1 April 2021, which is informed by a public relations newsletter and a website of the city. Additionally, it will be notified that women who underwent a hysterectomy cannot undergo the screening. Since the participants of this trial are a part of the target population for the screening, they can undergo routine screening. Routine screening will be performed at three community centres (on the bus) on 14 and 28 June, 2 and 12 July, 20 October, 10 November, and 3 December 2021, 


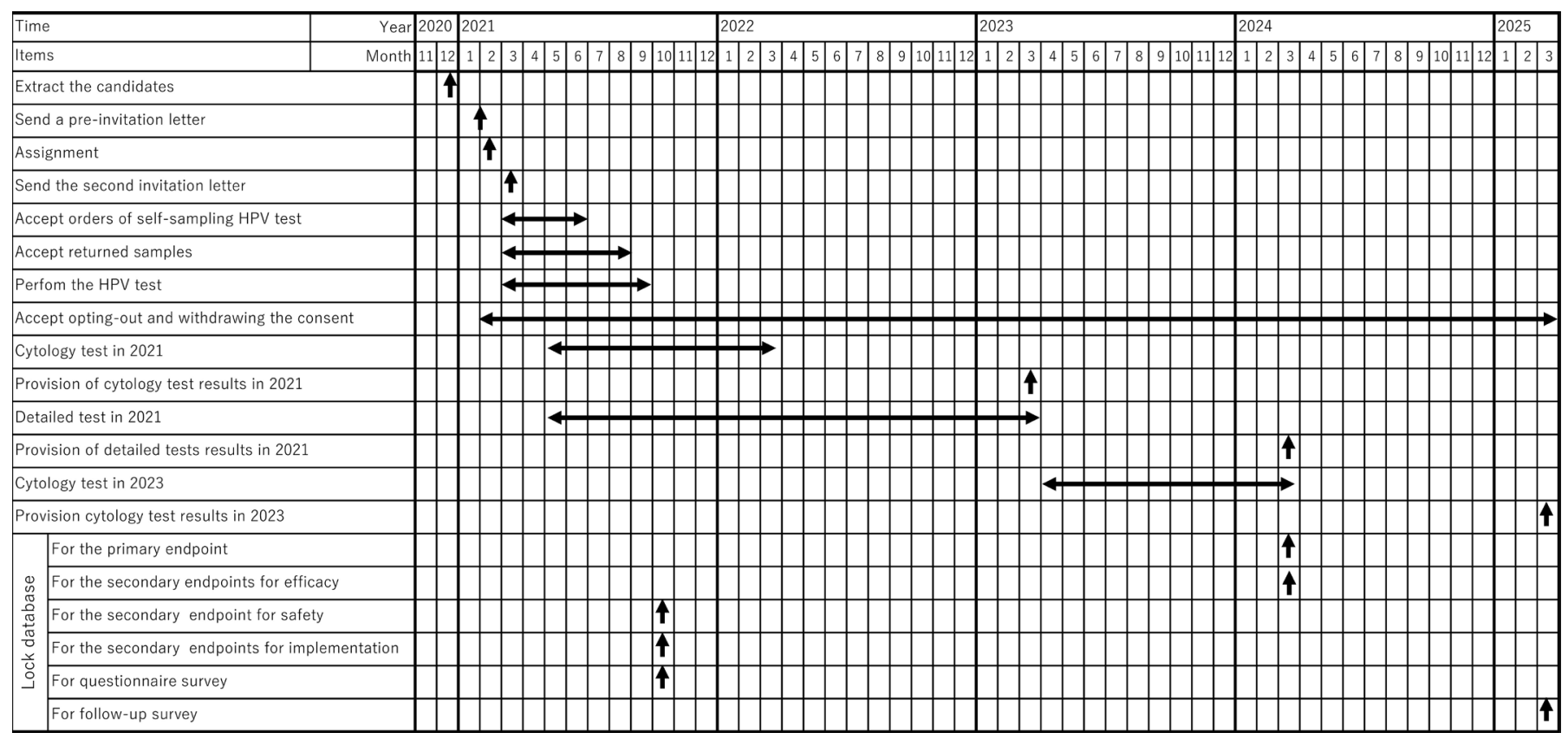

Figure 2 Time schedule plan. HPV, human papillomavirus.

and 17 and 20 January 2022. It will also be performed at the nine clinics in the city between 1 May 2021 and 31 March 2022. A sampling device for cytology tests will not be specified. Liquid-based cytology will be adopted in the screening at the community centres (on the bus) and in one clinic, and a conventional cytology test will be adopted in the remaining eight clinics. If the participants undergo screening at a community centre, the results of the cytology test will be sent from the health centre of the city. In contrast, if they undergo screening at clinics, the result will be reported by the clinic. If the result is atypical squamous cells of undetermined significance (ASC-US) or worse, the participants from health centres or clinics will be referred to some medical institutions for further testing using colposcopy and biopsy. The health centre of the city will collect the results of all cytology tests and almost all detailed tests (87.3\% in 2016) and administrate these data. The results of the cytology tests performed between 1 May 2021 and 31 March 2022, will be provided by the city to the Foundation by 31 March 2023, for this trial. The results of detailed tests performed by 31 March 2023, will be provided by 31 March 2024. Additionally, the data of whether the participants undergo cervical cancer screening in the fiscal year 2023 (between 1 April 2023 and 31 March 2024) will be provided by 31 March 2025.

\section{Self-sampling arm}

After assignment, the second invitation letter will be sent to the participants assigned to the self-sampling arm on 10 March 2021 (online supplemental file 3). The letter will indicate that they can choose screening with the selfsampling HPV test or routine screening test and how they can order the self-sampling kit, Evalyn Brush (Rovers Medical Devices, Oss, Netherlands). Although previous studies have reported no adverse event related to the use of this kit, ${ }^{7820}$ mild vaginal bleeding and pain might occur; however, at a low frequency. Additionally, the letter will inform the participants who have undergone a hysterectomy, are pregnant, and had no sexual experience that they cannot undergo self-sampling HPV testing. A kit order will be accepted by phone or via the website by 30 June 2021. On their orders, a self-sampling kit, an explanatory booklet, an informed consent form (online supplemental file 4), a questionnaire (online supplemental file 5), and an envelope for the sample return with cash on delivery will be sent. The explanatory booklet will provide instructions on how to collect a sample, how to fill the consent form, how to send a sample, the deadline for sending a sample and how to contact for a sample collection-related query. The participants will be asked to collect the sample at home and to return the sample, filled consent form and filled questionnaire. If the informed consent form is not returned to the Foundation, the HPV test and questionnaire survey will not be performed. To send the samples to the laboratory of an outsourced company (LSI Medience, Tokyo, Japan), a staff member of that company will pick up the samples daily. The samples will be stored at room temperature, the condition recommended by the kit manufacturer. The HPV test will be performed using the cobas 8800 system (Roche Diagnostics, Rotkreuz, Switzerland), in which the B-globin internal cellular control is detected to prevent false negatives. HPV-negative specimens with a negative $B$-globin result are flagged as invalid. The frequency of reported invalid results is rare. ${ }^{121316}$ When the HPV test result is invalid, the laboratory will perform retest up to two times. The HPV test results, that is positive, negative, or invalid, for each HPV type (type 16, type 18, and other 12 pooled high-risk HPV (hrHPV) including types 31, 33, 
$35,39,45,51,52,56,58,59,66$ and 68 ) will be reported to the Foundation from the laboratory, and we will send the results and cervical cancer screening information in the city to the participants by mail through an outsourced company (Accelight, Tokyo, Japan). For all participants who will undergo self-sampling HPV testing, we will recommend routine cervical cancer screening (cytology test) provided by the city. The messages for informing the participants of their results are shown below: hrHPVnegative: hrHPV was not detected in your sample. Thus, your risk for cervical cancer is thought to be low. However, the possibility of a false negative is not completely excluded. Additionally, the current guidelines for cervical cancer screening in Japan do not yet recommend the selfsampling HPV test. Therefore, we recommend that you undergo the routine cervical cancer screening involving the cytology test. Please carry this result notification along when you undergo the cytology test provided by the city because a doctor may refer to it.

hrHPV-positive: hrHPV was detected in your sample. You have a relatively high risk of developing cervical cancer. If high-risk types 16 and/or 18 were detected, the risk is thought to be higher. Be sure to undergo the routine cervical cancer screening involving the cytology test. Please carry this result notification when you undergo the cytology test provided by the city because a doctor may refer to it.

Procedures for routine screening (cytology test), detailed tests and providing the results will be the same as that for the control arm as mentioned above.

Although we will not give any information to the primary outcome assessors (doctors in the screening institutions), they will not be blinded completely because the participants who will undergo self-sampling HPV testing will carry the HPV test results along. The reason for adopting this strategy is that this procedure is expected to be used if the self-sampling HPV test is recommended as a screening option in future, thus providing real-world evidence.

\section{Time schedule plan}

This trial will be performed between 22 December 2020 and 31 March 2025 (figure 2).

- Selection and data extraction of candidates who meet the inclusion criteria: 22 December 2020.

- Registration of the candidates who meet the inclusion criteria: 23 December 2020.

- Sending a preinvitation letter: 1 February 2021.

- Enrolment and assignment: 22 February 2021.

- Sending the second invitation letter to the participants assigned to the self-sampling arm: 10 March 2021.

- Accepting orders for the self-sampling test: By 30 June 2021.

- Accepting samples from the participants: By 31 August 2021.

- Performing the HPV test: By 30 September 2021.
- Accepting opt-out and withdrawal of consent: By 31 March 2025.

- Cervical cancer screening in 2021 (cytology test): Between 1 May 2021 and 31 March 2022.

- Detailed tests based on the results of the cytology test in 2021: By 31 March 2023.

- Cervical cancer screening in 2023 (cytology test): Between 1 April 2023 and 31 March 2024.

- Database lock.

- Analyses of the primary and secondary outcomes for effectiveness: By 31 March 2024, including 'proportion of cytology tests performed.'

- Analysis for safety, implementation and questionnaire survey: By 31 October 2021, except for 'proportion of cytology tests performed.'

- Analysis for follow-up survey (screening uptake in 2023): By 31 March 2025.

\section{Endpoints}

Primary endpoint

The primary endpoint is the detection of CIN2 or worse determined by detailed tests (biopsy) conducted by 31 March 2023. CIN2 or worse will be included in the following situations:

- CIN2.

- CIN3.

- Adenocarcinoma in situ.

- Squamous cell carcinoma (SCC: including microinvasive and invasive SCG).

- Adenocarcinoma (including microinvasive and invasive adenocarcinoma).

- Adenosquamous carcinoma.

- Other cervical primary cancer.

- Metastatic cancer in the cervix.

Secondary endpoints for effectiveness

- Screening uptake.

- Participants who have received the cytology test.

- Participants who required detailed tests.

- Participants who have received detailed tests

- Detection of CIN2 or worse per cytology test.

- Positive predictive values.

Detailed definitions will be described in the statistical analysis plan (SAP), as shown in online supplemental file 6.

\section{Secondary endpoint for safety}

- Incidence of adverse events.

Detailed definitions will be described in the SAP (online supplemental file 6).

Endpoints aimed toward implementation

- Proportion of self-sampling HPV tests ordered.

- Proportion of self-sampling HPV tests ordered through the website.

- Proportion of self-sampling HPV tests returned.

- Proportion of positive HPV tests.

- Proportion of invalid HPV tests.

- Proportion of cytology tests received. 
- Time to each event.

- Time to ordering of self-sampling HPV tests.

- Time to sending of self-sampling kits to the participants.

- Time to return of self-sampling kits by the participants.

- Time to ordering of HPV test to the laboratory.

- Time to return of HPV test results by the laboratory.

- Time to sending of HPV test results to the participants.

- Total time.

Detailed definitions will be described in the SAP (online supplemental file 6).

\section{Questionnaire survey}

- Reasons for not having cervical cancer screening.

- Knowledge about HPV

- Experience of self-sampling HPV test.

- Preference for screening, sampling by a doctor, or self-sampling.

The questionnaire is shown in online supplemental file 5 , and detailed definitions will be described in the SAP (online supplemental file 6).

\section{Follow-up survey}

In the follow-up survey, screening uptake in 2023 will be compared between the two arms.

Detailed definitions will be described in the SAP (online supplemental file 6).

\section{Sample size calculation}

The sample size was calculated based on the primary outcome of detection of CIN2 or worse. According to previous studies, we set the following assumptions:

\section{Self-sampling arm}

Among the participants in the self-sampling arm, the proportion of those who will undergo self-sampling HPV testing (A): $0.13 .^{1316} 21-24$

In A, the proportion of HPV-positive participants (B): $0.117 .^{25}$

In $\mathrm{B}$, the proportion of participants who will undergo cytology testing $(\mathrm{C}): 0.79 .^{14-172126-29}$

In $\mathrm{C}$, the proportion of participants whose cytology test result is ASC-US or worse (D): $0.18 .^{25} 30$

In $\mathrm{D}$, the proportion of participants who will undergo detailed testing (E): 0.99. ${ }^{141528} 29$

In $\mathrm{E}$, the proportion of participants detected as CIN2 or worse (F): 0.54..$^{14} 15283132$

Among the participants in the self-sampling arm, the proportion of those who will undergo cytology testing without self-sampling HPV testing (G): 0.03. ${ }^{10} 132833$

In $G$, the proportion of participants whose cytology test result is ASC-US or worse $(\mathrm{H}): 0.03 .^{25} 30$

In $\mathrm{H}$, the proportion of participants who will undergo detailed testing (I): $0.74 .^{12} 28293435$

In I, the proportion of participants detected as CIN2 or worse $(\mathrm{J}): 0.34 . .^{12} 2829313234-36$
Control arm

- Among the participants in the control arm, the proportion of those who will undergo cytology test (K): $0.03 .{ }^{10132833}$

- In K, the proportion of participants whose cytology test result is ASC-US or worse (L): $0.03 .{ }^{25} 30$

- In L, the proportion of participants who will undergo detailed testing (M): 0.74. ${ }^{12} 28293435$

- In M, the proportion of the participants detected with CIN2 or worse lesions (N): 0.34. ${ }^{12} 282931$ 32 34-36

Based on these assumptions, the detection rate of CIN2 or worse lesions in each arm is calculated as follows:

Self-sampling arm: $\quad A * B * C * D * E * F+G * H$

$* \mathrm{I} * \mathrm{~J}=0.001156+0.000226=0.00138$

Control arm: $\mathrm{K} * \mathrm{~L} * \mathrm{M} * \mathrm{~N}=0.00023$

To detect the difference in the rate between the two arms under a two-sided alpha of 0.05 and power of 0.80 , the calculated sample size is 19094 . We assumed that $5 \%$ of women will refuse to participate in the trial (opting out) or have an incorrect address. Finally, the sample size should be approximately 20000 (10 000 in each arm).

The population of women in Ichihara City aged between 30 and 59 years as of 1 October 2020, was 48945. Since the city provides cervical cancer screening to only women with an even number of age, we divided the population by two $(48,945 / 2=24473)$. According to the Report on Regional Public Health Services and Health Promotion Services in 2018, the proportion of women who did not undergo cervical cancer screening for at least 2 years was $84 \%$. Thus, we multiplied the remaining number by 0.84 (24 $473 \times 0.84=20557)$. We estimated that 20557 women would meet the inclusion criteria of this trial.

\section{Population to be analysed}

Intention to screen

Intention to screen (ITS) will include all participants who are randomised, excluding those who will opt out after randomisation and those who withdraw their written consent.

\section{Modified ITS}

Modified ITS (mITS) will include all participants who are randomised, excluding those who will opt out after randomisation and those who will withdraw their written consent. However, in the endpoints, cytology tests and detailed tests in HPV-negative participants will not be included.

\section{Per protocol}

Per protocol (PP) will include all participants in the self-sampling arm who will undergo self-sampling HPV testing or cytology testing and the participants in the control arm who will undergo cytology testing. However, in the endpoints, cytology tests and detailed tests in HPVnegative participants will not be included.

Other population to be analysed

Details will be described in the SAP (online supplemental file 6$)$. 


\section{Analysis plan}

Primary endpoint analysis plan

The frequency and percentage with $95 \%$ CI will be calculated for each arm. The risk ratio and risk difference in the percentages of participants detected with CIN2 or worse between the two arms (risk ratio=percentage of participants detected with CIN2 or worse in the self-sampling $\mathrm{arm} /$ that in the control arm; risk difference=percentage of participants detected with CIN2 or worse in the selfsampling arm-that in the control arm) and the 95\% CI will be calculated. The test for the null hypothesis (the detection rate of CIN2 or worse is equal among the two arms) will be performed using Pearson's $\chi^{2}$ test. As necessary, sensitivity analyses using the Mantel-Haenszel test adjusted for the time period since the last screening (3-5 years, 6 years or more, those without a history) and age category ( $30-39$ years, $40-49$ years, $50-59$ years) will also be performed.

ITS will be used in the primary analysis as a population to be analysed. As necessary, sensitivity analyses will be performed using mITS and PP.

\section{Other analysis plan}

Details will be described in the SAP (online supplemental file 6$)$.

\section{Patient and public involvement statement}

The general population and women living in Ichihara City were not involved in preparing the study protocol, registration of participants and conducting the trial.

\section{ETHICS AND DISSEMINATION \\ Ethical considerations}

To ensure autonomous participation and reduction of selection bias as much as possible, opt-out consent will be obtained from all participants. In detail, we will send a preinvitation letter to all women who meet the inclusion criteria, with the option to opt out. Additionally, we will obtain written consent from the participants who undergo self-sampling HPV testing. The procedure complies with the Ethical Guidelines for Medical and Health Research Involving Human Subjects in Japan. This trial was approved by the Research Ethics Committees of the Chiba Foundation for Health Promotion and Disease Prevention (approval number R2-2), Graduate School of Medicine, Chiba University (approval number 3979) and Institute of Statistical Mathematics (approval number ISM20-001). Since Ichihara City does not have an ethics committee, the Committee of the Chiba Foundation for Health Promotion and Disease Prevention reviewed the protocol instead of Ichihara City (approval number R2-7).

For this trial, Ichihara City provided the existing data to the Foundation. Before the provision, the Committee for Personal Information Protection of Ichihara City reviewed the plan of this trial on 12 November 2020, and authorised data provision on 15 December 2020.
Considering this approval, the city was allowed to provide existing data and to launch this trial.

\section{Data sharing}

We will not share any data of this trial following the opinions of the Committee for Personal Information Protection of Ichihara City.

\section{Publication of the results}

The results from this trial will be disseminated through peer-reviewed journals and conference presentations after protecting the human rights of the subjects and their related parties and managing the interests of researchers and related parties. According to the statement from the International Committee of Medical Journal Editors, the authors will be determined.

\section{Author affiliations}

${ }^{1}$ Department of Health Research, Chiba Foundation for Health Promotion and Disease Prevention, Chiba, Japan

${ }^{2}$ Department of Public Health, Chiba University Graduate School of Medicine, Chiba, Japan

${ }^{3}$ Clinical Research Center, Chiba University Hospital, Chiba, Japan

${ }^{4}$ Research Center for Medical and Health Data Science, The Institute of Statistical Mathematics, Tachikawa, Tokyo, Japan

${ }^{5}$ Municipal Health Center, Department of Health and Welfare, Ichihara City, Ichihara, Japan

${ }^{6}$ Department of Reproductive Medicine, Chiba University Graduate School of

Medicine, Chiba, Japan

${ }^{7}$ Yushudai Clinic, Ichihara, Chiba, Japan

${ }^{8}$ Center for Preventive Medical Sciences, Chiba University, Chiba, Japan

Correction notice This article has been corrected since it was first published. The author affiliation section has been updated.

Acknowledgements We sincerely appreciate the invaluable support of Joji Koide, the mayor of Ichihara City. We also sincerely appreciate the advice of Yasushi Saito, Takehiko Fujisawa, and Yoshitaka Okamoto and the support of the staff members of the Chiba Foundation for Health Promotion and Disease Prevention, Chiori Suzuki, Fumika Kumahara, Fumiya Chiwaki, Hideaki Nagai, Ikumu Matsushita, Kenji Ishii, Makoto Koumi, Michiko Fusaeda, Saeri Omori, and Tsuyoshi Moritake. We would like to thank Editage (www.editage.com) for English language editing.

Contributors MF, TK and AH contributed to the study conception. MF, MShi, KN, MSu, IT, MSa, SY, MSho, HH, TK and AH contributed to the design of the trial. MF, MSu, IT, MSa, SY, NT and AH contributed to data acquisition. MF and AH wrote the draft of the study protocol and the manuscript. MF, MShi, KN, HH and AH revised the study protocol. All authors revised the manuscript. MF wrote the draft of the SAP and $\mathrm{KN}$ and $\mathrm{AH}$ revised it. All authors approved the manuscript to be published.

Funding This work was supported by JSPS KAKENHI Grant (http://www.jsps.go.jp/ j-grantsinaid, grant number 20H03906).

Disclaimer The funders had no role in study design, data collection and analysis, decision to publish, or preparation of the manuscript.

Competing interests None declared.

Patient consent for publication Not applicable.

Provenance and peer review Not commissioned; externally peer reviewed.

Supplemental material This content has been supplied by the author(s). It has not been vetted by BMJ Publishing Group Limited (BMJ) and may not have been peer-reviewed. Any opinions or recommendations discussed are solely those of the author(s) and are not endorsed by BMJ. BMJ disclaims all liability and responsibility arising from any reliance placed on the content. Where the content includes any translated material, BMJ does not warrant the accuracy and reliability of the translations (including but not limited to local regulations, clinical guidelines, terminology, drug names and drug dosages), and is not responsible for any error and/or omissions arising from translation and adaptation or otherwise. 
Open access This is an open access article distributed in accordance with the Creative Commons Attribution Non Commercial (CC BY-NC 4.0) license, which permits others to distribute, remix, adapt, build upon this work non-commercially, and license their derivative works on different terms, provided the original work is properly cited, appropriate credit is given, any changes made indicated, and the use is non-commercial. See: http://creativecommons.org/licenses/by-nc/4.0/.

\section{ORCID iDs}

Misuzu Fujita http://orcid.org/0000-0001-5308-4744

Kengo Nagashima http://orcid.org/0000-0003-4529-9045

\section{REFERENCES}

1 Hall MT, Simms KT, Lew J-B, et al. The projected timeframe until cervical cancer elimination in Australia: a modelling study. Lancet Public Health 2019;4:e19-27.

2 Hanley SJB, Yoshioka E, Ito Y, et al. HPV vaccination crisis in Japan. Lancet 2015;385:2571

3 OECD. OECD health statisics, 2019. Available: http://www.oecd.org/ els/health-systems/health-data.htm

4 Cancer Statitics in Japan. The editorial board of the cancer statistics in Japan, 2018. Available: https://ganjoho.jp/data/reg_stat/statistics/ brochure/2018/cancer_statistics_2018.pdf

5 Yagi A, Ueda Y, Kakuda M, et al. Epidemiologic and clinical analysis of cervical cancer using data from the population-based Osaka cancer registry. Cancer Res 2019;79:1252-9.

6 Yagi A, Ueda Y, Nakagawa S, et al. Potential for cervical cancer incidence and death resulting from Japan's current policy of prolonged suspension of its governmental recommendation of the HPV vaccine. Sci Rep 2020;10:15945.

7 Polman NJ, Ebisch RMF, Heideman DAM, et al. Performance of human papillomavirus testing on self-collected versus cliniciancollected samples for the detection of cervical intraepithelial neoplasia of grade 2 or worse: a randomised, paired screen-positive, non-inferiority trial. Lancet Oncol 2019;20:229-38.

8 Onuma T, Kurokawa T, Shinagawa A, et al. Evaluation of the concordance in HPV type between self- and physician-collected samples using a brush-based device and a PCR-based HPV DNA test in Japanese referred patients with abnormal cytology or HPV infection. Int J Clin Oncol 2020;25:1854-60.

9 Sultana F, Mullins R, English DR, et al. Women's experience with home-based self-sampling for human papillomavirus testing. BMC Cancer 2015;15:849.

10 Szarewski A, Cadman L, Mesher D, et al. HPV self-sampling as an alternative strategy in non-attenders for cervical screening - a randomised controlled trial. Br J Cancer 2011;104:915-20.

11 Bosgraaf RP, Ketelaars PJW, Verhoef VMJ, et al. Reasons for nonattendance to cervical screening and preferences for HPV selfsampling in Dutch women. Prev Med 2014;64:108-13.

12 Winer RL, Lin J, Tiro JA, et al. Effect of mailed human papillomavirus test kits vs usual care reminders on cervical cancer screening uptake, precancer detection, and treatment: a randomized clinical trial. JAMA Netw Open 2019;2:e1914729.

13 Elfström KM, Sundström K, Andersson S, et al. Increasing participation in cervical screening by targeting long-term nonattenders: randomized health services study. Int J Cancer 2019:145:3033-9.

14 Gök M, Heideman DAM, van Kemenade FJ, et al. HPV testing on self collected cervicovaginal lavage specimens as screening method for women who do not attend cervical screening: cohort study. BMJ 2010;340:c1040.

15 Gök M, van Kemenade FJ, Heideman DAM, et al. Experience with high-risk human papillomavirus testing on vaginal brush-based selfsamples of non-attendees of the cervical screening program. Int $J$ Cancer 2012;130:1128-35.

16 Tranberg M, Bech $\mathrm{BH}$, Blaakær J, et al. Preventing cervical cancer using HPV self-sampling: direct mailing of test-kits increases screening participation more than timely opt-in procedures - a randomized controlled trial. BMC Cancer 2018;18:273.

17 Haguenoer K, Sengchanh S, Gaudy-Graffin C, et al. Vaginal selfsampling is a cost-effective way to increase participation in a cervical cancer screening programme: a randomised trial. Br J Cancer 2014;111:2187-96.
18 Polman NJ, Snijders PJF, Kenter GG, et al. HPV-based cervical screening: rationale, expectations and future perspectives of the new Dutch screening programme. Prev Med 2019;119:108-17.

19 Pedersen K, Fogelberg S, Thamsborg LH, et al. An overview of cervical cancer epidemiology and prevention in Scandinavia. Acta Obstet Gynecol Scand 2018;97:795-807.

20 Yamasaki M, Abe S, Miura K. The effect of self-sampled HPV testing on participation in cervical cancer screening on a remote island. Acta Med Nagasaki 2018;62:55-61.

21 Kellen E, Benoy I, Vanden Broeck D, et al. A randomized, controlled trial of two strategies of offering the home-based HPV self-sampling test to non- participants in the Flemish cervical cancer screening program. Int J Cancer 2018;143:861-8.

22 Lam JUH, Rebolj M, Møller Ejegod D, et al. Human papillomavirus self-sampling for screening nonattenders: Opt-in pilot implementation with electronic communication platforms. Int $J$ Cancer 2017;140:2212-9.

23 Broberg G, Gyrd-Hansen D, Miao Jonasson J, et al. Increasing participation in cervical cancer screening: offering a HPV selftest to long-term non-attendees as part of RACOMIP, a Swedish randomized controlled trial. Int J Cancer 2014;134:10.1002/ ijc.28545:2223-30.

24 Ivanus U, Jerman T, Fokter AR, et al. Randomised trial of HPV selfsampling among non-attenders in the Slovenian cervical screening programme ZORA: comparing three different screening approaches. Radiol Oncol 2018:52:399-412.

25 Morisada T, Teramoto K, Takano H, et al. CITRUS, cervical cancer screening trial by randomization of HPV testing intervention for upcoming screening: design, methods and baseline data of 18,471 women. Cancer Epidemiol 2017;50:60-7.

26 Bais AG, van Kemenade FJ, Berkhof J, et al. Human papillomavirus testing on self-sampled cervicovaginal brushes: an effective alternative to protect nonresponders in cervical screening programs. Int J Cancer 2007;120:1505-10.

27 Darlin L, Borgfeldt C, Forslund O, et al. Comparison of use of vaginal HPV self-sampling and offering flexible appointments as strategies to reach long-term non-attending women in organized cervical screening. J Clin Virol 2013;58:155-60.

28 Sancho-Garnier H, Tamalet C, Halfon P, et al. HPV self-sampling or the Pap-smear: a randomized study among cervical screening nonattenders from lower socioeconomic groups in France. Int $J$ Cancer 2013;133:2681-7.

29 Cadman L, Wilkes S, Mansour D, et al. A randomized controlled trial in non-responders from Newcastle upon Tyne invited to return a self-sample for human papillomavirus testing versus repeat invitation for cervical screening. J Med Screen 2015;22:10.1177/0969141314558785:28-37.

30 Kasai T, Tachibana M, Kurokawa Y. Effectiveness of new technology (co-testing) combining liquide-based cytology and human papilloma virus DNA testing for mass screening of uterine cervical cancer. Chiba Survey Res J 2017;6:29-36.

31 Rijkaart DC, Berkhof J, Rozendaal L, et al. Human papillomavirus testing for the detection of high-grade cervical intraepithelial neoplasia and cancer: final results of the POBASCAM randomised controlled trial. Lancet Oncol 2012;13:78-88.

32 Kitchener HC, Almonte M, Thomson C, et al. HPV testing in combination with liquid-based cytology in primary cervical screening (ARTISTIC): a randomised controlled trial. Lancet Oncol 2009;10:672-82.

33 Jalili F, O'Conaill C, Templeton $\mathrm{K}$, et al. Assessing the impact of mailing self-sampling kits for human papillomavirus testing to unscreened non-responder women in Manitoba. Curr Oncol 2019;26:167-72.

34 Gustavsson I, Aarnio R, Berggrund M, et al. Randomised study shows that repeated self-sampling and HPV test has more than two-fold higher detection rate of women with CIN2+ histology than Pap smear cytology. Br J Cancer 2018;118:10.1038/ bjc. 2017.485:896-904.

35 Arrossi S, Thouyaret L, Herrero R, et al. Effect of self-collection of HPV DNA offered by community health workers at home visits on uptake of screening for cervical cancer (the EMA study): a population-based cluster-randomised trial. Lancet Glob Health 2015;3:e85-94.

36 Ronco G, Giorgi-Rossi P, Carozzi F, et al. Efficacy of human papillomavirus testing for the detection of invasive cervical cancers and cervical intraepithelial neoplasia: a randomised controlled trial. Lancet Oncol 2010;11:249-57. 